\title{
Optical properties of defective carbon nanotube $(7,7)$
}

\author{
S. A. Sozykin, V. P. Beskachko \\ South Ural State University, Chelabinsk, Russia \\ sozykinsa@susu.ru, beskachkovp@susu.ru
}

PACS 42.50.Ct

DOI 10.17586/2220-8054-2018-9-1-73-75

\begin{abstract}
The current article presents the results of a study of the effect of single-walled carbon nanotubes carcass defects on their electronic structures and optical properties. The study was carried out using an ab-initio quantum mechanical approach: the pseudo-potential method in the density functional theory (DFT) framework in the local density approximation. It is shown that the defects of a single or double vacancy, and StoneWales change the absorption spectrum of nanotubes. This can be expressed in the appearance of absorption in the low-energy region and in the smearing of the absorption peaks corresponding to electron transitions between Van Hove singularities near the Fermi energy.
\end{abstract}

Keywords: carbon nanotube, density of states, band structure, adsorption.

Received: 20 June 2017

Revised: 22 October 2017

\section{Introduction}

The electronic structure of carbon nanotubes (CNTs) has been studied theoretically in several papers using various methods and approximations. It has been shown that in the energy spectrum of a CNT there are a number of Van Hove singularities unique for each pair of chirality indices (n, m). Transitions between singularities symmetrically disposed about $E_{F}$, correspond to pronounced peaks in the absorption spectrum (transitions of the type $M_{i i}$ for CNTs with metallic conductivity, where $i$ belongs to the set of natural numbers). This allows us to use optical spectra to determine the chiral indices of CNTs. The paper [1] reports on the observation of characteristic absorption energies in 46 different CNTs. We can assume that the electronic structure and absorption spectra of perfect nanotubes have been studied quite well. At the same time, nanotubes can contain various defects in the structure of natural or artificial origin, which can influence the discussed properties of CNTs. In the literature, at present, data on this is insufficient to judge the observability of the corresponding effects and their use to identify defective structures. In this paper, we estimate the influence of defects on the electronic structure and the absorption spectrum of nanotubes with a metallic type of conductivity, using the methods of first-principle modelling.

\section{Model and method of modelling}

As the object of study, a single-walled CNT $(7,7)$ with the metallic type of conductivity and with the relatively small unit cell of 14 atoms was chosen. The model was a fragment of a perfect tube of 224 carbon atoms (16 elementary cells) with periodic boundary conditions. As shown in [2], the use of such "long" models is necessary for the correct modelling of CNTs containing defects. Given the size of the simulated cell, we chose the SIESTA package, which is not too demanding for computational resources, using the pseudo-potential formalism. The standard norm conserving Troullier-Martins pseudo-potentials are utilized. For exchange-correlation, potential local-density approximation given by D. M. Ceperley and B. J. Alder has been used. The absorption spectrum was calculated in the polarized mode, which is implied by the application of an electric field in " $z$ " direction (nanotube axis). A MeshCutoff of $200 \mathrm{Ry}$ is used for the grid to represent the charge density and the mesh of k-points for Brillouin zone integrations is $1 \times 1 \times 32$. We used finer mesh of $3 \times 3 \times 50 \mathrm{k}$-points for optical calculations. The structures are optimized by minimizing the forces on individual atoms (below $0.4 \mathrm{eV} / \mathrm{nm}$ ).

\section{Results}

In Fig. 1a we show the calculated band structure of the perfect CNT (7,7). It qualitatively coincides with the band structure given in the paper [3]. In the vicinity of the Fermi level at $\sim 2 \mathrm{eV}$ DOS is nonzero and almost constant. This region corresponds to a linear dispersion in the band structure. It would seem that in the presence of unoccupied states directly above the Fermi energy, absorption must be observed in the low-energy region. This, however, does not occur (see Fig. 2a): there is no absorption up to previously mentioned $2 \mathrm{eV}$. A similar arrangement of the peaks $M_{11}$ and $M_{22}$ have also been experimentally [4]. As noted in the paper [5], the optical transition between the highest occupied molecular orbital and lowest unoccupied molecular orbital sub-bands in 
armchair CNTs is symmetry forbidden. It is shown in Fig. 2a that the parameters $M_{11}, M_{22}$ and $M_{33}$ were equal to $2.11 \mathrm{eV}, 3.34 \mathrm{eV}$ and $3.77 \mathrm{eV}$, respectively. The following values [6] of these parameters were obtained in the full-potential method in the WIEN2k package: $2.2 \mathrm{eV}, 3.5 \mathrm{eV}$ and $4.0 \mathrm{eV}$. The result for $M_{11}$ obtained in [6] slightly agrees with experiment $(2.43 \mathrm{eV}$ [1]) than the result of this work, but this agreement remains at a level of $10 \%$.

In Fig. 1(b-f), it is shown how the band structure and DOS of a nanotube change when defects of the StoneWales type and vacancies appear on it. The presence of a single vacancy leads to an increase in the density of states immediately above the Fermi level and a change in the behavior of DOS near the Van Hove singularities (Fig. 1b). Due to a change in DOS near the Fermi level, absorption appears in the low-energy region. The corresponding peak in Fig. 2b is comparable in magnitude with the more diffuse, and one and a half times less intense compared to defect-free nanotubes peaks $M_{11}, M_{22}$ and $M_{33}$.
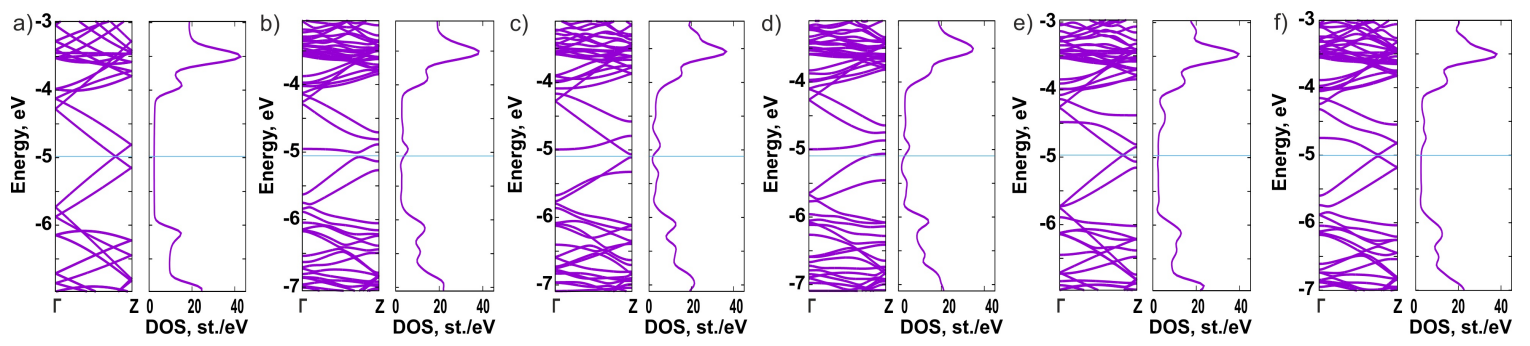

FIG. 1. Band structure and electronic structure (density of states) of defect-free (perfect) CNTs $(7,7)$ (a) and CNTs $(7,7)$ with defects: single vacancy (b); Double vacancies in orientations of $n$ (c) and $t$ (d); Stone-Wales defects in $n$ (e) and $t$ (f) orientations. The horizontal line shows the position of the Fermi level

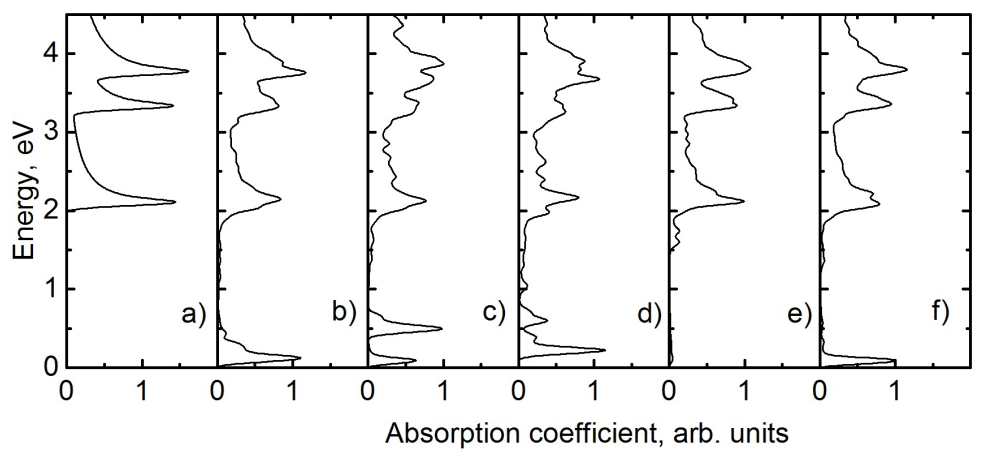

FIG. 2. The absorption spectra of defect-free (a) and nanotubes with defects of single (b), double vacancies in orientations of $n(\mathrm{c})$ and $t(\mathrm{~d})$, Stone-Wales defects in $n(\mathrm{e})$ and $t(\mathrm{f})$ orientations

In the energy spectrum of CNTs with defects of a double vacancy of type $2 \mathrm{Vn}$ and $2 \mathrm{Vt}$, there is a significant number of valence states above and below the Fermi level (Fig. 1c,d), between which the transition is not prohibited. This leads to the appearance of two peaks in the absorption spectra in the energy range up to $1 \mathrm{eV}$ (Fig. 2c,d).

In the case of a Stone-Wales defect of the first type (in the n-orientation), DOS in the vicinity of the Fermi level is noticeably higher than that of a defect-free nanotube (Fig. 1e). This leads to the appearance of an absorption peak in the energy region of less than $0.25 \mathrm{eV}$ (Fig. 2e). The introduction of a first type (n-orientation) Stone-Wales defect into the CNT structure increases DOS only above the Fermi level (Fig. 1e). This does not lead to a change in the absorption spectrum in the low-energy region (Fig. 2e).

It is seen that for all considered types there are five dispersion curves near the $\mathrm{Z}$ point in the band structure. The appearance of the additional curve cannot be associated with localized charges on the defect. When using the chosen basis set that could occur when the length of model less than $1 \mathrm{~nm}$, but in our work, this length was $2 \mathrm{~nm}$.

A common feature of the absorption spectra of defective nanotubes, according to the results obtained by us, is the smearing of the characteristic peaks $M_{11}, M_{22}$ and $M_{33}$ in the absence of noticeable displacements of these peaks. 


\section{Conclusion}

Thus, the article presents information on the effect of the most common defects on the electronic structure and the absorption spectrum of a carbon nanotube with an initially metallic type of conductivity. It was found that the defects considered do not lead to the appearance of a forbidden band in the energy spectrum of CNTs $(7,7)$. Differences in the nature of the spectra (the presence-absence of one or two absorption peaks in the low-energy part of the spectrum, as well as the degree of blurring of peaks $M_{11}, M_{22}$ and $M_{33}$ ) indicate the possibility of identifying the type of defect by empirical means.

\section{Acknowledgements}

The work was supported by Act 211 Government of the Russian Federation, contract No. 02.A03.21.0011.

\section{References}

[1] Fantini C., Jorio A., Souza M., Strano M., Dresselhaus M., Pimenta M. Optical Transition Energies for Carbon Nanotubes from Resonant Raman Spectroscopy: Environment and Temperature Effects. Phys. Rev. Lett., 2004, 93(14), P. 147406.

[2] Sozykin S.A., Beskachko V.P., Vyatkin G.P. Calculation of Adsorption Parameters for Lithium on Carbon Nanotube (7,7) with a Double Vacancy. Mater. Sci. Forum, 2016, 843, P. 132-138.

[3] Su W.-S. Electronic and structural properties of carbon nanotubes modulated by external strain. J. Appl. Phys., 2013, 113, P. 244308.

[4] Matea A., Baibarac M., Baltog I. Optical properties of single-walled carbon nanotubes highly separated in semiconducting and metallic tubes functionalized with poly(vinylidene fluoride). J. Mol. Structure, 2017, 1130, P. 38-45.

[5] Malić E., Hirtschulz M., Milde F., Knorr A., Reich S. Analytical approach to optical absorption in carbon nanotubes. Phys. Rev. B, 2006, 74(19), P. 195431.

[6] Gharbavi K., Badehian H. Optical properties of armchair (7,7) single walled carbon nanotubes. AIP Advances, $2015,5(7)$, P. 77155. 